\title{
Propuesta para el desarrollo de una aplicación para la gestión administrativa en la UNED, Costa Rica
}

\author{
Ana Gabriela Bejarano Salazar ${ }^{1}$ \\ Viviana Berrocal Carvajal ${ }^{2}$ \\ lleana Salas Campos ${ }^{3}$ \\ Cinthya Valerio Álvarez ${ }^{4}$
}

\author{
1. Programa de Informática Educativa, UNED; abejarano@uned.ac.cr \\ 2. Programa de Informática Educativa, UNED; vberrocal@uned.ac.cr \\ 3. Programa de Producción Electrónica Multimedial, UNED; isalas@uned.ac.cr \\ 4. Programa de Aprendizaje en Línea,UNED; cvalerio@uned.ac.cr
}

Recibido: 15 de octubre del 2014
Aceptado: 11 de diciembre del 2014

\begin{abstract}
Resumen
El artículo presenta los resultados de la investigación sobre los servicios que debe brindar la aplicación para dispositivos móviles que facilite la gestión administrativa de profesores y estudiantes de la Universidad Estatal a Distancia (UNED) de Costa Rica. La primera fase de la metodología consistió en una revisión de las aplicaciones institucionales para dispositivos móviles que utilizan algunas universidades nacionales e internacionales. Los resultados obtenidos mostraron que $100 \%$ poseen el servicio de noticias y el mapa del campus universitario, $83,3 \%$ ofrecen los horarios de clases o tutorías y el acceso a redes sociales, $66,7 \%$ la opción para ingresar a la biblioteca, la lista de eventos, la información de carreas y las plataformas en línea. Luego, en una segunda fase, se aplicó un cuestionario auto administrado a estudiantes y profesores de la UNED, con el fin de conocer la tenencia de dispositivos móviles y las preferencias de servicios que podría brindar una aplicación para móviles en la UNED. Los resultados indicaron que $75 \%$ de los estudiantes que tienen teléfono celular, acceden a Internet a través de ellos, $30 \%$ manifestó tener tabletas y $72 \%$ computadoras portátiles. Tanto profesores como estudiantes señalaron que las preferencias de servicios accesibles desde dispositivos móviles son, en orden de prioridad: matrícula, acceso a Blackboard y Moodle, información de carreras, horario de tutorías y la posibilidad de enviar correos.
\end{abstract}

\section{Abstract \\ Proposal for the development an administrative management app at the UNED, Costa Rica}

This paper presents the results of research services must provide the mobile application in order to facilitate the administrative management of teachers and students of the Universidad Estatal a Distancia (UNED) in Costa Rica. The first stage of the methodology consisted of a review of corporate applications for mobile devices using some national and international universities. The results showed that $100 \%$ own news service and map of the university campus, an $83.3 \%$ offer class schedules or tutorials and access to social networks, the $66.7 \%$ have a choice to enter the library, the list of events, information of education programs and online platforms. Then, in a second stage, a self-administered interview was applied to students and teachers of UNED, in order to know what kind of mobile devices they had and what services them preferred in a mobile application in the UNED. The results indicated that $75 \%$ of students that have cell phones, access to Internet through them, a $30 \%$ of them reported having tablets and a $72 \%$ laptops. Both teachers and students reported that the preferences of services accessible from mobile devices are in order of priority: tuition, access to Blackboard and Moodle, career information, schedule hours of tutorials and the ability to send emails. Further research could measure the impact 
Investigaciones posteriores podrían medir el impacto de la implementación de una aplicación institucional para dispositivos móviles en la UNED.

Palabras clave: Aprendizaje móvil, aplicación informática, programa informático didáctico, dispositivos móviles, comunicación móvil. of the implementation of an institutional application for mobile devices at UNED.

Key words: Mobile Learning, computer applications, educational software, mobile devices, mobile communication.

\section{INTRODUCCIÓN}

Actualmente, la expansión en la tenencia de dispositivos móviles está provocando una continua reflexión en las instituciones educativas, sobre su potencial para brindar ofertas académicas y replantearse los enfoques tradicionales, a la luz de propuestas más oportunas y ubicuas. Nuevas formas de relacionarse, compartir, comunicar, interactuar y transmitir la información fuerzan a reflexionar cómo hacer uso de la capacidad de estos dispositivos para los aspectos pedagógicos, así también para asuntos de gestión administrativa.

Por lo anterior, cada vez son más las casas de estudio que se suman a la tendencia de ofrecer a la comunidad universitaria aplicaciones institucionales desde donde brindan acceso a los servicios básicos: horarios de clase, calendario de actividades académicas, deportivas y culturales, agenda telefónica, matrícula y solicitud de certificaciones, entre las más comunes. Es decir la aplicación tiene una finalidad corporativa, ya que se orienta a los servicios administrativos más que a los académicos.

Este documento se centrará en la propuesta de un $a p p$ para la gestión administrativa y el propósito es mostrar los resultados de la investigación llevada a cabo para conocer no sólo la tenencia y tipo de dispositivos móviles en estudiantes y profesores de la UNED de Costa Rica, sino también sus preferencias en cuanto a los servicios qué debería brindar la universidad, a través de una aplicación nativa que sea descargable en su aparato electrónico.

Los resultados que se muestran forman parte de un proyecto mayor que busca el diseño de propuesta técnico-pedagógica para la implementación del aprendizaje móvil en la UNED.

\section{- Definición de app}

La palabra app se usa de manera informal como el diminutivo de application program. El término siempre ha sido utilizado por la comunidad de informáticos, sin embargo, según indica la revista PC Magazine (2012), con la creación de la tienda iTunes de Apple, en el año 2008, se ha popularizado y extendido su uso.

Por otra parte, el Diccionario de Oxford (2014) define app como una aplicación de software diseñada con un propósito particular, generalmente pequeña, especialmente creada para que pueda ser descargada en dispositivos móviles. Las apps tienen propósitos específicos y pueden ser desarrolladas para ejecutarse en diferentes sistemas operativos (Android, iOS, Symbian, Windows, WebOS). El usuario debe acceder a la tienda virtual (App Store, Ovi, Play Store, Windows Phone) del sistema operativo correspondiente a su dispositivo con el fin de buscar, descargar y actualizar aplicaciones.

Según lo señaló Koekkoek (2011), en el Informe de Distimo el año 2011, se caracterizó por un crecimiento exponencial del desarrollo y publicación de apps para dispositivos móviles. Más de un millón de aplicaciones fueron ofertadas, dadas las crecientes demandas de los usuarios para disponer de mayores funcionalidades y productos.

De acuerdo con el Reporte Horizon (2012), en el año 2013 las tendencias estarían enfocadas a las 
aplicaciones para dispositivos móviles y el uso de las tabletas. Según este reporte estos dos temas serían en ese momento parte de la vida cotidiana de las personas, y las universidades estarían desarrollando recursos para aprovecharlos.

En el campo educativo, y a partir de la investigación realizada, las autoras de este artículo distinguieron tres tipos de apps: las institucionales, orientadas a la gestión administrativa; las que brindan acceso a las plataformas Learning Management System (LMS) y las de contenido didáctico pedagógico. Este artículo se concentra en las características deseables de una app del primer tipo para la UNED.

\section{- Diferencia entre app nativa y web app}

Una app nativa es aquella que se elabora para un sistema operativo específico de un dispositivo móvil, tal como: Android, Blackberry, iOS, Windows, entre otros. Las ventajas del uso de este tipo de apps es que permiten un acceso a todas las funcionalidades del dispositivo (cámara, GPS, notificaciones, entre otros), se puede descargar la aplicación de la tienda específica (appstore) y la actualización es constante. En contraposición a estos beneficios, este tipo de desarrollo necesita más conocimiento en lenguajes de programación, no se puede reutilizar el código desarrollado para usarlo con otro sistema operativo y es más onerosa su producción (ver tabla 1).

Una web app es la aplicación que se programa en lenguajes que son independientes del sistema operativo del dispositivo móvil, de forma tal que es considerado un desarrollo multiplataforma. Las ventajas de este desarrollo es la reutilización del código, así resulta más económica su inversión, no necesitan estar colocadas en una tienda específica y se aprovechan los sitios web que están diseñados bajo este formato. No obstante, el desarrollar de esta manera implica no contar con acceso a las características del dispositivo (cámara, GPS, notificaciones, etc), siempre se requiere de conexión a Internet, el tiempo de respuesta de los servicios puede ser mayor que la $a p p$ nativa y al no estar visible en la tienda necesita de mucha promoción y esfuerzo para que los usuarios la utilicen (ver tabla 1).

Para contrastar estos desarrollos, la tabla 2 muestra la secuencia de eventos para acceder al app nativa de Stanford University. Al ingresar al entorno de la universidad puede observarse que en la $a p p$ nativa todos los servicios están concentrados en una misma pantalla y se observa cómo la interfaz ha sido modificada para facilitar la navegación en dispositivos como teléfonos inteligentes y tabletas. Por ejemplo: las noticias, los eventos y los videos se categorizaron y su presentación difiere de la visualización de la página web en un computador de escritorio.

TABLA 1

Comparación de las posibilidades entre una app nativa y una web app

\begin{tabular}{|c|c|c|}
\hline Criterios & App nativa & Web app \\
\hline Acceso a los recursos del dispositivo (cámara, GPS, notificaciones, entre otros). & Sí & No \\
\hline Siempre se requiere de conexión a Internet. & No & Sí \\
\hline Descargable desde la tienda (App Store, Ovi, Play Store, Windows Phone). & Sí & No \\
\hline Reutilización de código. & No & Sí \\
\hline $\begin{array}{l}\text { Para su desarrollo se requiere de conocimientos específicos (plataformas para el desarrollo } \\
\text { del sistema operativo) de lenguajes de programación. }\end{array}$ & Sí & No \\
\hline Permite la multiplataforma. & No & Sí \\
\hline Permite la actualización constante. & Sí & Sí \\
\hline Requiere promoción y divulgación para su uso desde dispositivos móviles. & Sí & Sí \\
\hline
\end{tabular}

Fuente: Elaboración propia. 
TABLA 2

Eventos para acceder a los servicios de Stanford University desde el app

\section{Descripción del evento}

\section{Evento 1:}

Imagen de acceso a la página principal de Stanford University

\section{Evento 2:}

Visualización de los servicios institucionales

\section{Evento 3:}

Visualización del calendario
Imagen desde dispositivo móvil (web nativa)

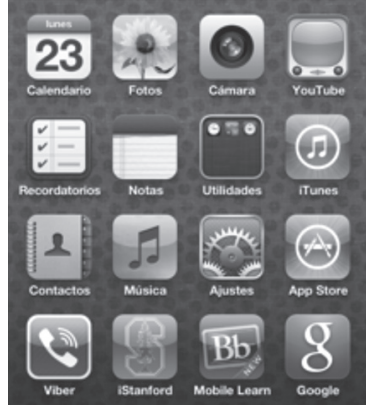

Tomado de Stanford University (2014)
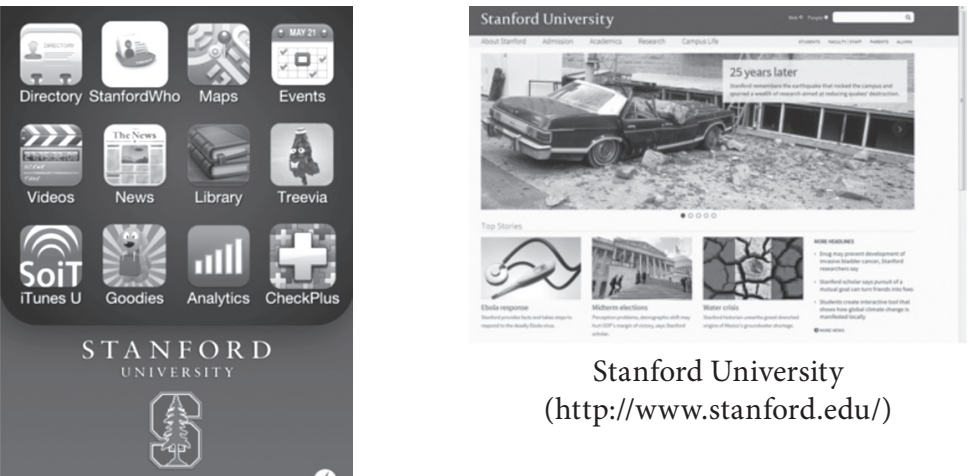

Stanford University (http://www.stanford.edu/)

Tomado de Stanford University (2014)

\begin{tabular}{ll}
\hline Atras Calendarios \\
\hline Q Buscar en todo contenido \\
\hline Todo & $>$ \\
\hline \multirow{1}{*}{ My Calendar } & $>$ \\
\hline Arts & $>$ \\
\hline Lecture / Reading & $>$ \\
\hline Clase & $>$ \\
\hline Health / Wellness & $>$ \\
\hline Tour & International
\end{tabular}

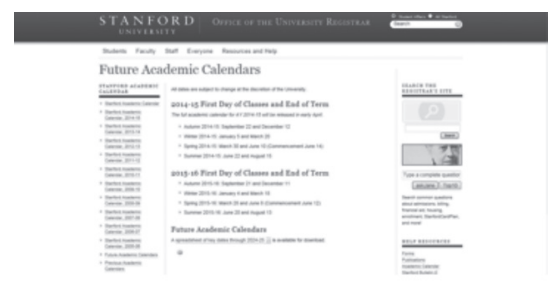

Stanford University (http://www.stanford.edu/)

Tomado de Stanford University (2014) 
TABLA 2 (Continuación)

\section{Descripción del evento}

\section{Evento 4:}

Visualización de eventos incluidos en el calendario de artes
Imagen desde dispositivo móvil (web nativa)

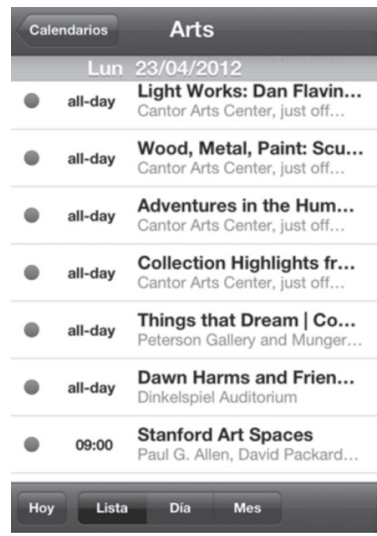

Tomado de Stanford University (2014)

Evento 5:

Visualización de noticia Things that Dream desde un smartphone
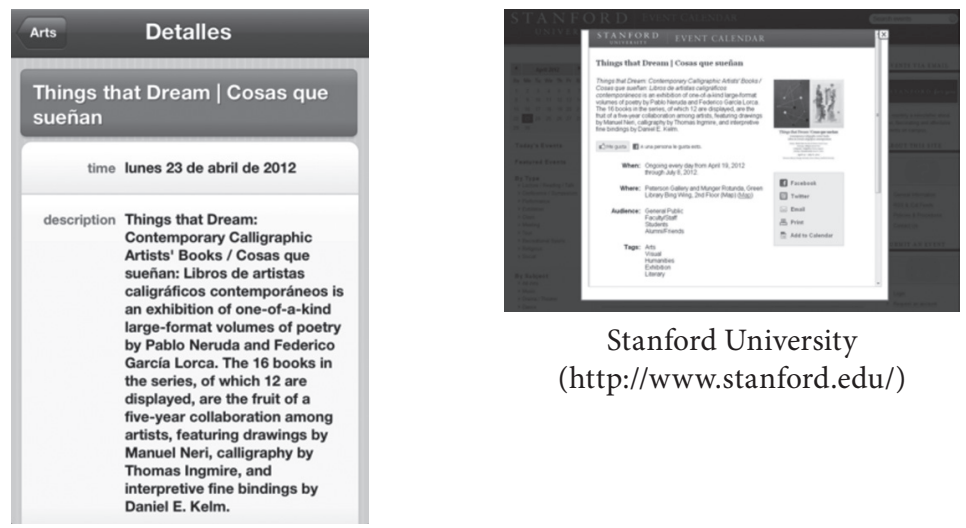

Stanford University (http://www.stanford.edu/)

Tomado de Stanford University (2014)

Fuente: App Stanford University y la Web.

\section{Sistemas operativos para dispositivos móviles}

El sistema operativo es, según Beekman (2011), un conjunto de programas instalados en un computador o en un dispositivo electrónico, tal como las tabletas y los teléfonos inteligentes, que tienen como objetivo llevar a cabo operaciones técnicas, entre ellas la comunicación con dispositivos periféricos (mouse, teclado, impresora, etcétera) y tareas más complejas de comunicación y seguridad. Beekman indica que el sistema operativo determina las aplicaciones de software que son compatibles con el hardware empleado, por esta razón es importante conocer cuáles son los sistemas operativos más utilizados en dispositivos móviles.

Sistema operativo iOS. Es el sistema operativo de Apple Inc. Este es un software privado que solo puede utilizarse en dispositivos diseñados por esta empresa tales como: iPhone, iPad, iPod. Salió a la luz pública en el año 2007. Apple acostumbra liberar frecuentemente una actualización del sistema operativo, lo que hace que los usuarios tengan la posibilidad de aprovechar cada vez más sus aparatos electrónicos.

Sistema operativo Android. Fue diseñado por la empresa Android Inc., posteriormente adquirida por Google en el año 2007, exclusivamente para 
dispositivos móviles. A diferencia del iOS es de código abierto, por lo tanto puede adaptarse a diferentes tipos de dispositivos. Esta posibilidad ha generado que muchas empresas, tales como LG, Samsung, HTC y otras, lo adoptaran y adecuaran para generar mayores posibilidades en sus aparatos.

Un aspecto importante por mencionar es que el único celular que utiliza el sistema operativo Android en su versión regular es el Nexus, lanzado al mercado por la empresa Google en convenio con LG.

Sistema operativo Windows Phone. Este sistema operativo le pertenece a la empresa Microsoft, es relativamente nuevo ya que su presentación en el mercado se realizó en el año 2010. El predecesor de este sistema fue el Windows Mobile, que no tuvo mucho éxito entre los usuarios móviles por estar enfocado hacia la población empresarial.

Existen muchas empresas que han optado por colocar este sistema en sus dispositivos móviles, entre ellas Nokia en sus teléfonos Lumia, Samsung en sus modelos Omnia M y W, los ZTE en sus celulares Orbit y Tania, Huawei y HTC.

\section{METODOLOGÍA}

La primera etapa de la investigación fue exploratoria, ya que identificó, mediante una revisión de las aplicaciones web de algunas universidades, el tipo de servicios que brindan tanto a estudiantes como a profesores para proporcionar información que sustente la propuesta de diseño de una aplicación nativa, descargable en dispositivos móviles, para la UNED. Las apps de las universidades analizadas pertenecen a la Universidad Hispanoamericana y la Universidad Fidélitas, en Costa Rica; la Universidad de Harvard, la Universidad de Stanford y la Nova Southeastern University en Estados Unidos y la Universidad Nacional de Educación a Distancia (UNED) de España.

La segunda fase es de tipo descriptiva, por cuanto aplica el uso de estadísticas derivadas de las frecuencias, en términos de incidencias o concordancias, en la preferencia de los usuarios (estudiantes y profesores) sobre el tipo de servicios que se deben ofrecer en una aplicación nativa para la gestión administrativa de la UNED. Para obtener la información se aplicó un cuestionario auto administrado a estudiantes y profesores de la universidad. El objetivo fue conocer la tenencia de dispositivos móviles y las preferencias de servicios.

En cuanto a la muestra, se eligió por conveniencia a docentes y estudiantes matriculados en los cursos virtuales que ofreció la UNED durante el primer cuatrimestre de 2014.

\section{RESULTADOS}

Esta sección se ha divido en dos apartados: en una primera parte expone los resultados de la comparación de las apps de las diversas universidades para apoyar la gestión administrativa; posteriormente, presenta los resultados de la aplicación del cuestionario a docentes y estudiantes relacionados con la tenencia de dispositivos móviles y la preferencia de servicios.

\section{- Comparación de servicios ofrecidos en las apps nativas de las universidades analizadas}

La tabla 3 muestra los resultados de la revisión de servicios ofrecidos a través de las apps nativas de la Universidad Hispanoamericana, la Universidad Fidélitas, la Universidad de Harvard, la Universidad de Stanford, la Nova Southeastern University y la Universidad Nacional de Educación a Distancia (UNED - España).

Una vez realizada la comparación de las apps se obtienen los siguientes resultados:

- El $100 \%$ de las universidades brindan acceso a las noticias y mapas institucionales. Algunas universidades clasifican las noticias por categorías, facultades, intereses, entre otros. El acceso a las redes sociales más usadas por su población estudiantil y publicación de horarios es importante para $83,3 \%$ de las universidades. Un $66,7 \%$ de las apps nativas brindan el acceso a la biblioteca, la información de carreras, 
TABLA 3

Resumen de los servicios que se pueden obtener de las apps institucionales

\begin{tabular}{|c|c|c|c|c|c|c|c|}
\hline Criterios & $\mathbf{H}$ & $\mathbf{F}$ & HD & $\mathbf{U}$ & ST & $\mathbf{N}$ & Porcentaje \\
\hline Acceso a la biblioteca & $\bullet$ & & $\bullet$ & & $\bullet$ & - & $66,7 \%$ \\
\hline Matrícula & - & & & & & - & $33,3 \%$ \\
\hline Radio & - & & & & & - & $33,3 \%$ \\
\hline Acceso a redes sociales & - & - & - & - & - & & $83,3 \%$ \\
\hline Compartir aplicación & - & & & & & & $16,7 \%$ \\
\hline Ubicación /mapa & - & - & - & - & - & - & $100 \%$ \\
\hline Envío de correos & $\bullet$ & & & - & & & $33,3 \%$ \\
\hline Eventos & & - & - & & - & - & $66,7 \%$ \\
\hline Carreras & & - & - & & - & - & $66,7 \%$ \\
\hline Noticias & - & - & - & - & - & - & $100 \%$ \\
\hline Horarios & • & - & • & • & • & & $83,3 \%$ \\
\hline Acceso a plataformas & & - & - & & - & - & $66,7 \%$ \\
\hline Directorio telefónico & & & - & & - & & $33,3 \%$ \\
\hline Horario de buses & & & - & & & - & $33,3 \%$ \\
\hline Comedores & & & • & & & - & $33,3 \%$ \\
\hline Número de emergencia & & & - & & - & - & $50 \%$ \\
\hline
\end{tabular}

Fuente: Elaboración propia a partir de la revisión de las apps de cada una de las universidades (H: Hispanoamericana; F: Fidélitas; HD: Harvard; U: UNED; ST: Stanford; N: Nova)

los eventos y las plataformas LMS del campus. Los eventos también pueden ser categorizados por intereses. En el caso de las plataformas LMS (Moodle y Blackboard Learn), algunas universidades muestran este servicio de manera independiente al de los sistemas de teleconferencia (Blackboard Collaborate, como en el caso de Nova Southeastern University).

- $\quad$ El 50\% de las universidades cuenta con sistemas de alerta o emergencias para los estudiantes y profesores.

Otros aspectos que incluyen las apps son: videos, radio, elaboración de contenidos educativos tanto de tutores como de estudiantes, admisiones, galerías fotográficas, directorios, preguntas frecuentes, solicitud de certificaciones y récord académico, entre otros.

\section{- Resultados de la aplicación del cuestionario a docentes y estudiantes relacionados con la tenencia de dispositivos móviles y preferencias de una app nativa}

En la aplicación del cuestionario auto administrado se contó con la respuesta de 211 docentes y 877 estudiantes representativos de todas las instancias académicas de la institución. El 65\% de los encuestados residen en la zona urbana de la Gran Área Metropolitana (GAM). Las personas que viven fuera de la GAM corresponden al 35\% (ver tabla 4).

Los siguientes apartados sintetizan, en términos porcentuales, la tenencia de los dispositivos móviles personales que poseen los profesores y estudiantes, así como la expectativa de servicios para un app nativo institucional. 
TABLA 4

Distribución de población encuestada por provincias

\begin{tabular}{lcc}
\multicolumn{1}{c}{ Provincia } & Zona urbana GAM & Zona rural \\
\hline Alajuela & $12,4 \%$ & $5,4 \%$ \\
Cartago & $12,4 \%$ & $2,8 \%$ \\
\hline Heredia & $10,1 \%$ & $1,4 \%$ \\
San José & $30,0 \%$ & $9,3 \%$ \\
Guanacaste & - & $5,8 \%$ \\
Puntarenas & - & $7,0 \%$ \\
Limón & - & $3,2 \%$
\end{tabular}

Fuente: Encuesta a estudiantes y funcionarios UNED, I cuatrimestre 2014

\section{- Tenencia de dispositivos móviles}

En cuanto a la tenencia de dispositivos móviles se obtuvieron los siguientes resultados: del total de encuestados $90 \%$ poseen teléfonos móviles, de ellos $63,3 \%$ un smartphone. Del total de personas con teléfonos móviles $75 \%$ tienen acceso a Internet.

Otros dispositivos con los que cuentan los estudiantes son: computadora portátil, reportado por $72 \%$ de los encuestados; las tabletas en $30 \%$ y la tenencia de reproductores de audio y video está indicada en $42 \%$.

Las marcas de dispositivos más empleados son los Samsung (45\%), Nokia (18\%), iPhone (13\%) y otras marcas mencionadas son Sony, Huawei, LG, Alcatel, Blackberry, entre otros (24\%). Esta información revela que los dispositivos empleados por los estudiantes poseen mayoritariamente el sistema operativo Android y en menor grado iOS, Symbian, y Blackberry.
Para conocer las intenciones en una eventual futura adquisición de dispositivos, por parte de estudiantes y profesores, se consultó a los encuestados la posibilidad de invertir en su compra. Así 46\% indicó que comprarían de manera prioritaria una computadora portátil, 29\% invertiría en un smartphone, mientras que solo $9 \%$ compraría una tableta y 1,5\% un reproductor de audio y video (ver tabla 5).

De lo anterior se infiere que las personas prefieren comprar computadoras con el objeto de apoyar sus procesos de estudio (57\%), pero que la eventual compra de dispositivos como smartphone (20\%) y tabletas (37\%), son opciones por considerar en la vinculación con la universidad a través de aplicaciones que permitan su uso para realizar diferentes gestiones (ver tabla 5).

Preferencia de servicios requeridos por profesores y estudiantes de la UNED en el app institucional. En cuanto a los servicios elegidos por los estudiantes y profesores para integrar en el app institucional de la UNED, el grupo de investigación brindó 19 servicios derivados de la primera etapa de esta investigación. La Tabla 6 muestra una distribución relativa de los servcios que podrían tener una app institucional, de acuerdo con la preferencia de estudiantes y profesores de la UNED.

De los datos registrados en la tabla 6 , se observa que la preferencia de los encuestados en cuanto a servicios prioritarios que se deben incluir en una app institucional de la UNED recae en los referentes a matrícula $(35,1 \%)$ y al ingreso a las plataformas LMS (23,1\%), como servicio de mayor demanda. Ambos coincidentes con los servicios que brindan las universidades analizadas en la primera parte de la investigación (ver tabla 3).

TABLA 5

Prioridad de compra de dispositivos móviles y razones de adquisición, según los encuestados

\begin{tabular}{|c|c|c|c|c|c|}
\hline \multirow{2}{*}{ Dispositivo } & \multirow{2}{*}{ Prioridad de compra } & \multicolumn{3}{|c|}{ Razón de adquisición } & \multirow{2}{*}{ NR } \\
\hline & & Estudio & Trabajo & Recreación & \\
\hline Computadora portátil & $46 \%$ & $57 \%$ & $28 \%$ & $1 \%$ & $14 \%$ \\
\hline Smartphone & $29 \%$ & $20 \%$ & $38 \%$ & $29 \%$ & $13 \%$ \\
\hline Tableta & $9 \%$ & $37 \%$ & $17 \%$ & $29 \%$ & $17 \%$ \\
\hline Reproductor de audio & $1,5 \%$ & $6 \%$ & $4 \%$ & $70 \%$ & $20 \%$ \\
\hline
\end{tabular}


Los servicios de noticias y los horarios tuvieron bajos niveles de preferencia por parte de los profesores y estudiantes (ver tabla 6). Sin embargo, en la tabla 3 es posible observar que son recurrentes en la mayoría de las universidades que cuentan con apps nativas.

En referencia al acceso a los servicos de la biblioteca, es necesario señalar que los estudiantes y los profesores de la UNED no le atribuyeron importancia para ser intergrado en una app nativa, solo $0,6 \%$, contrario a la fuerte tendencia que se visualiza en las apps de las universidades nacionales e internacionales analizadas (ver tabla 3). Por otra parte, destacan en la tabla 6 la consulta a información de carreras, horarios de examenes y tutorías, así como el acceso a los recursos didácticos.

Como opción adicional se les habilitó a los estudiantes la posibilidad de indicar cuáles otros servicios sugierían para integrar en una app institucional. De esta consulta se obtuvo un total de 427 respuestas que fueron clasificadas en servicios académicos y administrativos.

Dentro de los académicos fueron mencionados como prioritarios el mantener una comunicación sincrónica y asíncronica, el acceso a la oferta académica con anticipación, el acceso a tutorías y videotutorías, además de la consulta de documentos en el repositorio. En cuanto a los servicios administrativos, señalaron la consulta del historial académico y calificaciones, el pago de servicios (matrícula, exámenes de reposición, seguro estudiantil, trámites, entre otros), la información de centros universitarios sobre aulas de tutoría y aplicación de exámenes y la comunicación con la defensoría de los estudiantes.

Asociado a las respuestas brindadas por los estudiantes, también se les habilitó la posibilidad de emitir su criterio a los tutores sobre qué tipo de servicios adicionales solicitarían en una $a p p$ nativa institucional. Se obtuvo un total de 138 respuestas abiertas, las cuales fueron categorizadas en servicios académicos y administrativos para apoyar su gestión. Los resultados obtenidos muestran una preferencia por mantener una comunicación sincrónica y asincrónica con los estudiantes, el acceso a los recursos didácticos (audiolibros, libros digitales, podcast,
TABLA 6

Preferencia de servicios para una app UNED por parte de estudiantes y profesores

\begin{tabular}{lc}
\hline \multicolumn{1}{c}{ Servicios } & Porcentaje \\
\hline Matrícula & $35,1 \%$ \\
\hline Acceso a Blackboard y Moodle & $23,1 \%$ \\
\hline No responde & $15,6 \%$ \\
\hline Carreras de grado y posgrado & $6,1 \%$ \\
\hline Envío de correos & $4,3 \%$ \\
\hline Horarios de tutorías & $3,3 \%$ \\
\hline Horario de exámenes & $2,8 \%$ \\
\hline Acceso a recursos didácticos & $2,8 \%$ \\
\hline Orientaciones académicas & $1,4 \%$ \\
\hline Récord académico & $1,3 \%$ \\
\hline Aula donde se aplican los exámenes & $0,7 \%$ \\
\hline Preguntas frecuentes & $0,7 \%$ \\
\hline Acceso a la biblioteca & $0,6 \%$ \\
\hline Ubicación de los centros universitarios & $0,5 \%$ \\
\hline Noticias & $0,5 \%$ \\
\hline Directorio telefónico & $0,5 \%$ \\
\hline Eventos & $0,3 \%$ \\
\hline Acceso a redes sociales & $0,2 \%$ \\
\hline Horarios de videoconferencias & $0,1 \%$ \\
\hline Radio & $0,0 \%$ \\
\hline
\end{tabular}

Fuente: Encuesta a estudiantes y funcionarios UNED, I cuatrimestre 2014.

videos, objetos de aprendizaje), la posibilidad de visualizar videoconferencias, la consulta de la lista de estudiantes matriculados en curso e información de contacto; lo anterior en el área académica. En cuanto al área administrativa solicitan poder realizar trámites de recursos humanos (pago de viáticos, vacaciones, salario, nombramientos, evaluación del desempeño, entre otros) a través de los dispositivos móviles, la visualización la oferta de capacitaciones, la posibilidad de mantener una comunicación sincrónica y asincrónica con las cátedras, direcciones de escuelas y compañeros tutores.

Al realizar las consultas y clasificar las demandas se evidenció que 58,5\% de los servicios proporcionados por los estudiantes son del área administrativa, de igual manera $54,3 \%$ de los servicios señalados por los tutores. 


\section{CONCLUSIONES}

En relación con la tenencia, por parte de profesores y estudiantes de la UNED, de dispositivos así como de las posibilidades de acceso a Internet que facilitan, se considera justificado que la UNED invierta recursos económicos y humanos en el desarrollo de una app nativa institucional. Las funcionalidades de los dispositivos móviles para ampliar y mejorar la comunicación entre la universidad, estudiantes y profesores, para distribuir información y para realizar gestiones de manera más expedita son los argumentos más fuertes que apoyan la generación de la $a p p$ citada. Algunas de las razones que fundamentan los argumento son:

Para los estudiantes de la GAM una $a p p$ nativa puede ayudar a ubicar las aulas y laboratorios dentro del centro univeristario, mediante el GPS. Lo anterior sin descartar a los estudiantes de las zonas rurales que necesitan movilizarse a centros universitarios de otras regiones y podrían usar la opción de mapas, incluida en una app institucional que muestre la ubicación de los centros universitarios y las aulas.

Al descargar una app nativa en el dispotivo móvil se facilita el trabajo con la información integrada en la aplicación, sin conectividad a Internet.

Las validaciones por medio de las appstore promueven un desarrollo robusto en el sistema operativo del dispositivo, lo que garantiza una mayor calidad del producto.

Los informes de Horizon (2012) y Distimo (2011) señalan que hay una tendencia creciente de uso de apps por parte de los usuarios y por tanto un incremento en las búsquedas de este tipo de recursos en las tiendas especializadas.

Una app nativa permitiría concentrar varios servicios en un solo "clic", lo cual facilita la navegación del usuario.

Una app para la UNED debe considerar un desarrollo que responda a las características del sistema operativo que poseen los estudiantes. Tal como se manifestó en esta investigación un alto porcentaje posee dispositivos que funcionan con Android, sin embargo un porcentaje significativo emplean aparatos que funcionan con iOS, aspecto que no debe omitirse.

De manera prioritaria, según se desprende de los resultados obtenidos, los servicios que debe brindar una $a p p$ nativa de la UNED son: el acceso a los sistemas de matrícula, a las plataformas LMS, las consultas a los historiales académicos, el pago de servicios y certificaciones, la información de centros universitarios, especificamente aulas de tutorías y aplicación de exámenes. El acceso a los recursos didácticos fue señalado por los profesores como prioritario, así como la posibilidad de realizar consultas y trámites propios de sus nombramientos en la universidad.

Si bien algunos otros servicios no fueron considerados como prioritarios, son parte de la comunicación constante que debe asegurar una institución de educación superior con la comunidad académica, por eso, y partiendo de la revisión de las apps de las universidades nacionales e internacionales, se puede desprender que servicios de noticias, calendarios, eventos y hasta de emisión de alertas de emergencias, no pueden ser excluidos.

Por otra parte, es menester señalar el bajo porcentaje que refleja la tabla 6 , en contraste con la tabla 4, en la opción de acceso a los servicios de la biblioteca. Es este sentido se podrían desprender dos hipótesis, que pueden analizarse de manera independiente o interrelacionada. La primera es que el sistema de biblioteca no brinda suficientes servicios a los estudiantes y los profesores como para satisfacer sus necesidades de información donde quiera que ellos se encuentren. Esto es, más allá del solo acceso a bases de datos, dar servicios de búsqueda de documentos en bases de datos externas, de préstamo y envío de libros a domicilio, entre otros muy propios de bibliotecas de instituciones educativas con modelos a distancia. La segunda hipótesis está relacionada con la exigencia que la UNED hace a sus estudiantes y profesores de emplear intensamente los recursos que posee la biblioteca, tanto físicos como digitales, de modo que no se considere como un recurso indispensable. Algunas recomendaciones:

- Capacitar al personal de desarrollo de software de la Dirección de Tecnología, Información 
y Comunicaciones de la UNED en el uso frameworks de los diferentes sistemas operativos para dispositivos móviles.

- Diseñar y desarrollar una aplicación nativa para la UNED, tanto para Android como para iOS, que en una primera versión contenga algunos de los servicios considerados prioritarios por estudiantes y profesores.

- Realizar una prueba piloto de una primera versión de app nativa institucional para la UNED.

- Valorar los resultados de la prueba piloto, partiendo de un estudio que se realice con el Centro de Investigación y Evaluación Institucional (CIEI) con el fin de validar el diseño y el desarrollo de la app.

- Publicar en las tiendas especializadas las apps desarrolladas para los sistemas operativos Android e iOS.

\section{REFERENCIAS}

Beekman, G. (2011). Introducción a la computación. México: Pearson Educación de México S.A.

Harvard University. (2014). Harvard mobile (4.4) [Mobile application software]. Recuperado de http:// store. apple.com/0075s?afid=p238|bqJ64t4F-dc_ mtid_1870765e38482_pcrid_5169198099_

Johnson, L., Adams, S., y Cummins, M. (2012). The NMC Horizon Report: 2012 Higher Education Edition. Austin, Texas: The New Media Consortium.
Koekkoek, H. (2011). Distimo Publication Full Year 2011. Recuperado de Distimo Publication: http:// www.distimo.com/download/publication/ Distimo_Publication_-_Full_Year_2011/EN/ archive/

Oxford. (2014). App. En Oxford Dictionaries. Recuperado de http://www.oxforddictionaries.com/ us/definition/american_english/app

PC Magazine. (2012). App. En PC Magazine. Recuperado de: http://www.pcmag.com/ encyclopedia/term/37865/app

Nova Southeastern University. (2014). iShark (3.8) [Mobile application software]. Recuperado de http://store.apple.com/us?afid=p238|bqJ64t4F-dc_ mtid_1870765e38482_pcrid_5169198099_

Stanford University. (2014). iStanford (3.1) [Mobile application software]. Recuperado de http:// store.apple.com/us?afid=p238|bqJ64t4F-dc_ mtid_1870765e38482_pcrid_5169198099_

Universidad Fidélitas. (2014). Universidad Fidélitas (3.5) [Mobile application software]. Recuperado de http://store.apple.com/us?afid=p238|bqJ64t4F-dc mtid_1870765e38482_pcrid_5169198099_

Universidad Hispanoamericana. (2014). Hispanoamericana lógica digital (3.2) [Mobile application software]. Recuperado de http:// store.apple.com/us?afid=p238|bqJ64t4F-dc_ mtid_1870765e38482_pcrid_5169198099_

UNED Cervera. (2014). UNED Cervera (1.0.0) [Mobile application software]. Recuperado de http:// store.apple.com/us?afid=p238|bqJ64t4F-dc_ mtid_1870765e38482_pcrid_5169198099 
